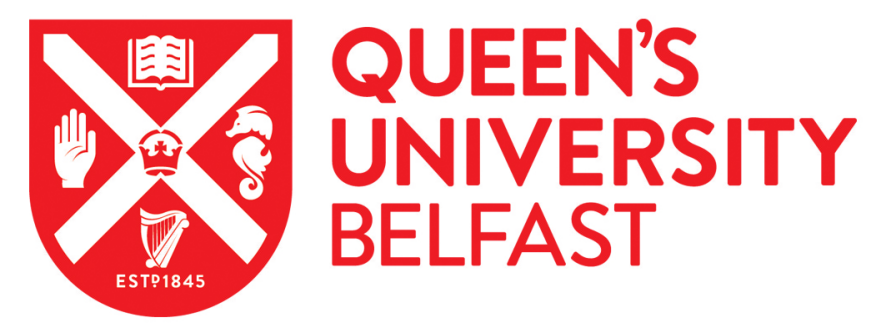

\title{
CAD/CAM milled removable complete dentures: time and cost estimation study
}

Srinivasan, M., Schimmel, M., Naharro, M., O'Neill, C., McKenna, G., \& Muller, F. (2019). CAD/CAM milled removable complete dentures: time and cost estimation study. Journal of Dentistry, 80, 75-79.

https://doi.org/10.1016/j.jdent.2018.09.003

Published in:

Journal of Dentistry

Document Version:

Peer reviewed version

Queen's University Belfast - Research Portal:

Link to publication record in Queen's University Belfast Research Portal

Publisher rights

Copyright 2018 Elsevier Ltd

This manuscript is distributed under a Creative Commons Attribution-NonCommercial-NoDerivs License

(https://creativecommons.org/licenses/by-nc-nd/4.0/), which permits distribution and reproduction for non-commercial purposes, provided the author and source are cited

\section{General rights}

Copyright for the publications made accessible via the Queen's University Belfast Research Portal is retained by the author(s) and / or other copyright owners and it is a condition of accessing these publications that users recognise and abide by the legal requirements associated with these rights.

Take down policy

The Research Portal is Queen's institutional repository that provides access to Queen's research output. Every effort has been made to ensure that content in the Research Portal does not infringe any person's rights, or applicable UK laws. If you discover content in the Research Portal that you believe breaches copyright or violates any law, please contact openaccess@qub.ac.uk. 


\section{Introduction}

Conventional fabrication protocols for the construction of complete removable dental prostheses (CRDPs) are well established and continue to form part of contemporary clinical practice [1]. These conventional complete denture protocols (CCDPs) require multiple patient visits with considerable amounts of chairside- and laboratory time. The conventional protocol is considered the current 'gold-standard' for undergraduate teaching.

Recent developments have facilitated the introduction of computer aided design and manufacturing $(\mathrm{CAD} / \mathrm{CAM})$ technologies into the fabrication of CRDPs. The initial attempts to manufacture CRDPs with CAD/CAM methods began in the 1990's [2, 3]. A steady evolution and improvement of the technologies, has led to an exponential increase in the number of providers and systems available in the market today [4]. Evidence suggests that CRDPs manufactured by CAD/CAM methods are equivalent or superior to conventionally manufactured CRDPs in terms of accuracy [5-9] and have improved material properties [10, 11]. A number of studies have demonstrated very high levels of patient satisfaction with CRDPs constructed with CAD/CAM methods [12-14]. The introduction of CAD/CAM technology revolutionizes both the manufacturing processes for CRDPs and the clinical protocols required for denture construction.

This is evidenced by the two-visit digital denture protocol (DDP) which reduces considerably the number of patient visits and employs new clinical materials $[15,16]$. The DDP utilises specialised stock impression trays, elastomeric impression materials, specific instruments for recording vertical dimensions and centric relations. This does require a small amount of specialised clinical training for the clinical operators. In comparison, the CCDP employs traditional low-cost materials, instruments that are universally available and requires no additional training for experienced operators. 
Before incorporating new protocols into clinical practice and/or teaching curricula consideration must be given to patient safety and satisfaction, efficacy of the procedures and the economic costs involved. Whilst it is imperative to continue to adopt newer and more effective clinical methods to ensure best possible therapeutic outcomes in patient care, it is equally important to quantify the economic feasibility of such novel interventions.

Therefore, the aim of this quality improvement project was to compare the economic costs of the two-visit DDP with the traditional CCDP in the undergraduate student clinic of the University of Geneva, Switzerland [17]. Costs were measured from the perspective of a university-setting care provider and included: clinical chairside time, materials, and laboratory fees. The null hypotheses for this study stated that there would be no differences in costs incurred or constructing CRDPs when following either the DDP or the CCDP.

\section{Materials and Methods}

Twelve undergraduate final year dental students in the Division of Gerodontology, at the University Clinics of Dental Medicine, University of Geneva constructed CDs for their respective patients. The undergraduates had received didactic teaching in removable prosthodontics but had no prior denture construction experience. The students constructed a first set of CRDPs following the CCDP as described in the undergraduate clinical curriculum. They then constructed a second set of CRDPs for the same patients using the two-visit DDP. Six of the twelve students constructed a single upper CD (Group\#1) opposing a full or partial antagonistic dentition while the remaining six students constructed both upper and lower CDs (Group \#2) for completely edentulous patients. The procedures included were part of the ongoing undergraduate teaching programme at the University of Geneva. The protocol for this study was submitted to the local ethics committee and as a "quality improvement project" was deemed exempt from formal ethical approval. 


\subsection{Two-visit Digital Denture protocol (DDP)}

The two-visit DDP (AVADENT, Global Dental Science, Scottsdale, AZ, USA) followed by the students for constructing the CAD/CAM milled CRDPs, is described in detail in a previously published report [15]. The DDP comprised two patient visits. The first visit involved capturing all the clinical records required for denture construction and then a subsequent second visit for denture insertion. At the first visit, master impressions of the edentulous arches were made with prefabricated stock impression trays, using polyvinylsiloxane (PVS) impression material (AVADENT ${ }^{\mathrm{TM}}$, Global Dental Science Europe BV, Tilburg, The Netherlands). The inter-occlusal jaw relationship was recorded using an anatomical measuring device (AVADENT ${ }^{\mathrm{TM}}$, Global Dental Science Europe BV, Tilburg, The Netherlands) and was confirmed with a gothic arch tracing using the same device. The jaw record was registered using a PVS bite registration material (AVADENT ${ }^{\mathrm{TM}}$, Global Dental Science Europe BV, Tilburg, The Netherlands). The anterior plane of occlusion was aligned parallel to the inter-pupillary line using a special occlusal plane indicator supplied by the manufacturer (AVADENT ${ }^{\mathrm{TM}}$, Global Dental Science Europe BV, Tilburg, The Netherlands). The aesthetic parameters (labial fullness, labial support, lip and smile lines) were then verified and tooth selection (size, form, and shade) completed. The clinical records and patient photographs were sent to the digital denture laboratory with a completed prescription form.

The digital lab technician scanned all the clinical records and imported them into purpose-built design software (AVADENT ${ }^{\mathrm{TM}}$, Global Dental Science Europe BV, Tilburg, The Netherlands). The anatomical landmarks were identified and the peripheral limits were set on the virtual models. The latter were then aligned according to the clinically captured jaw relation records, and a virtual teeth set-up was performed. This virtual set-up was sent as 
an electronic preview to the clinician for approval. A subsequent preview was generated after incorporation of any changes suggested by the clinician. Once the final preview was approved the CRDPs were milled, finished, and shipped to the clinic. At the second patient visit the dentures were inserted and post-operative instructions provided.

\subsection{Conventional Complete Denture Protocol (CCDP):}

The CCDP followed involved 5-6 patient appointments. At the first visit, a preliminary impression with an irreversible hydrocolloid impression material (DENTSPLY DeTrey $\mathrm{GmbH}$, Konstanz, Germany) was made, using stock Schreinemakers trays for edentulous impressions [18]. This impression was used to pour a preliminary stone cast for the fabrication of an auto-polymerizing polymethylmethacrylate (PMMA) resin custom impression tray in a conventional dental laboratory. At the second visit, the custom tray was checked for fit and extension followed by border moulding using ADA specification type-1 low fusing impression compound (Kerr Dental Europe, Bioggio, Switzerland). Master impressions were taken with zinc oxide eugenol impression paste (SS White, Gloucester, England) and sent to the laboratory for fabrication of master models. Permanent base plates were constructed on these models and occlusal wax rims were added. The third visit involved a face bow transfer, recording of vertical and horizontal jaw relations, aesthetic parameters, and tooth selection. The centric jaw relation record was verified with a conventional gothic arch tracing using intra-oral tracers. The models were mounted in the laboratory and anterior tooth setup completed. A fourth clinical visit served to verify the anterior tooth setup intraorally. Once complete, the articulator was returned to the technician for posterior tooth setup. The fifth visit was for the final try-in of the completed set-up. The final dentures were fabricated using heat polymerizing polymethylmethacrylate resin, trimmed and polished by the dental technician. The sixth and final clinical visit was for denture insertion. 


\subsection{Outcome Measures}

\subsubsection{Clinical Chairside Time}

The chairside time spent by the student for each patient visit during the entire course of denture construction was timed with a stopwatch and recorded in minutes for each of the two denture protocols. A single investigator $\left(\mathrm{MS}^{1}\right)$ clocked the students discretely and none of the students were aware of the time being noted or for what purpose. The total time spent, in minutes, was then calculated combining all the visits together for each denture protocol. A mean time spent by each student for each protocol was then calculated for each of the two groups.

\subsubsection{Costs: Clinical fee, Clinical materials, and Laboratory costs}

Costs were divided into clinical fee, clinical materials, and laboratory costs. The clinical fees were the costs the dental school charges the patient for the clinical steps, and this is billed at a $40 \%$ discounted rate. This cost is inclusive of the clinical materials but exclusive of the dental laboratory fees. This is a fixed cost by the institution and was uniformly generated for all patients irrespective of the denture protocol.

Clinical materials costings were generated for all materials used on the clinic during the two denture protocols. The material costs comprised of expenditure incurred during each of the two clinical protocols (including impression materials, trays and instruments used). However, inclusion of infrastructure costs was beyond the scope of this work. The laboratory costs were taken from the final laboratory invoice billed for the completed denture work.

\subsubsection{Cost minimization analysis}

Based on the clinical costs charged by the institution, an estimated hourly labour cost was calculated. The estimated hourly labour cost was calculated using the formula given below:

$$
\text { Estimated hourly labour cost }=\frac{\text { clinical cost }- \text { mean } \text { clinical materials cost }}{\text { mean } \text { chairside time }(\text { in hours })}
$$

This formula was applied to group\#1 and group\#2, with the university discount. 


\subsection{Statistical analysis}

Data was verified for normal distribution and paired t-tests were used for statistical analysis. The level of statistical significances was set to $p<0.05$. All statistical analyses were done using a statistical software package Statistica ver.12 (Dell, Tulsa, OK, USA). This is a pilot analysis prior to a planned RCT, so no sample size was done. In case of a non-significant outcome, post hoc power analysis was planned to report the possibility of any type-II errors [19].

\section{Results}

The mean chairside time spent, calculated clinical materials cost, laboratory costs incurred and the calculated overall costs for the institution (clinical materials + laboratory costs) are shown in Table 1.

\subsection{Clinical chairside time spent and costs}

The mean chairside time spent, calculated clinical materials cost, laboratory costs incurred and the calculated overall costs for the institution (clinical materials + laboratory fees) are shown in Table 1. CCDP required significantly longer clinical time than the DDP for both Group\#1 $(\mathrm{p}=0.0206)$ and Group\#2 $(\mathrm{p}=0.0020)$. The clinical materials costs were higher for the DDP for both (Groups\#1 $\mathrm{p}<0.0001$; Group\#2: $\mathrm{p}=0.0002$ ). The overall costs (clinical materials + laboratory costs), were significantly higher for the CCDP than the DDP (Group\#1: $\mathrm{p}=0.0032 ;$ Group\#2: $\mathrm{p}=0.0080$ ).

\subsection{Cost minimization analysis}

A cost minimization analysis was conducted as the outcome of the two construction protocols yielded the same results i.e. fabrication of CRDPs. Table 2 illustrates that the DDP protocol 
is the least costly alternative for construction of CRDPs. By extrapolating this result to a minimum of one set of fitted dentures by the undergraduate students during their curriculum in the Division of Gerodontology, at the University Clinics of Dental Medicine, at the University of Geneva in Switzerland, a significant cost savings of over 145,000 Swiss francs (approx. €125,000/-) could be generated over a 10-year period.

\section{Discussion}

Digital technologies are already contributing to clinical dentistry. Whilst CCDPs are still widely taught in dental schools around the world, DDP may become the norm for denture fabrication in the future. Therefore, in order to adequately train dental students, the DDP techniques will have to be taught in dental schools. However before implementing this new teaching protocol in the undergraduate curriculum an effective quality improvement project needs to be completed. Only after having established the feasibility of the DDP protocol and its non-inferiority compared to the CCDP, should this be incorporated into the curriculum. From a budgetary standpoint it is also imperative that the costs involved in the new teaching protocol are thoroughly examined.

Economic evaluation can be divided into four types: cost-minimization; cost-effectiveness; cost-utility and cost-benefit analyses. Cost minimization can be applied to appraisal of medical treatments when the outcome of alternative procedures has been demonstrated to be the same. A cost minimization analysis simply focuses on finding the least costly alternative. It does not appraise individual outcomes or quantify the benefits of alternative approaches. This study simply compared the costs generated from two methods of constructing CRDPs: the traditional CCDP and the DDP and so this analysis was appropriate to answer the research question. However, the consequences of the two alternatives, in terms of costs for 
maintenance and follow-up visits, were not explored or measured in this study. Whilst this is a limitation of this work, this consideration was beyond the scope of this initial project.

CAD/CAM milled CRDPs are a novel treatment in removable prosthodontics. This study represents the first, which was designed to explore the economic costs involved in their fabrication compared with traditional approaches.

The study sample was small and taken from an undergraduate student clinic. However, CAD/CAM milled prostheses are not widely used in settings outside university hospitals so generating a similar sample in other environments would have been extremely challenging. In this study, the average chairside times recorded were longer than what has been previously reported in former studies assessing similar outcomes [20]. This may be explained by a number of reasons. Firstly, it has to be borne in mind that the treatments performed in previous studies were executed by experienced prosthodontists with a good deal of experience in denture fabrication methods [20]. In the current study the treatments were performed by undergraduate students, who had very limited experience in constructing complete dentures and under supervision. This inexperience contributes to the length of the clinical procedure. Moreover, as per university clinic protocols, every clinical step is checked and verified by a clinical instructor. Sometimes, the steps would have to be repeated multiple times before being certified as clinically acceptable; yet another factor that would add to the chairside time spent. However, it is safe to assume, that the chairside time would be considerably lower (and potentially less costly) for experienced clinicians for both clinical protocols. The data gathered in this study clearly illustrates that in Switzerland, the DDP is a less costly method of producing CRDPs. It must be borne in mind that clinical and laboratory costs are high in Switzerland and these may not represent equivalent figures from other countries. As illustrated from the cost minimization analysis, adoption of the DDP in 
this particular hospital could generate substantial economic savings if scaled up to include all clinical cases.

As the oral health of the adult population has evolved, clinicians are faced with fewer completely edentate cases. Such cases can be challenging to manage as they tend to be concentrated amongst the oldest members of society. Given that the DDP requires significantly fewer appointments for patients and clinical time is much less, this approach may be more acceptable to patients and may represent better value for money if costs were measured from a societal perspective. All of these factors should be considered in more detail if the DDP is to become a more widely available treatment alternative for patients in a variety of clinical environments. Economic aspects are surely a major part of clinical decision making. This project assumed an equal outcome for both procedures, a complete denture. However, in a clinical setting, other factors like patient satisfaction, aesthetics, biocompatibility as well as maintenance and repair may nuance the similarity of the outcome of both procedures. The DDP protocol is in its infancy stage and more research is needed for a variety of aspects which should be considered in clinical decision making.

\section{Conclusions}

In the Division of Gerodontology, at the University Clinics of Dental Medicine, University of Geneva, the DDP is less costly compared with the CCDP. The costs for clinical chairside time and laboratory costs were significantly less although materials costs were higher. Based on these findings the null hypotheses for this study is rejected as there are differences in costs incurred for constructing CRDPs utilising the DDP compared to the CCDP. 


\section{Declaration of interests}

Conflicts of interest: none

\section{Source of funding}

This research did not receive any specific grant from funding agencies in the public, commercial, or not-for-profit sectors. This cost estimation study was entirely funded by institutional funds of the Division of Gerodontology and Removable Prosthodontics, University Clinics of Dental Medicine, University of Geneva, Geneva, Switzerland and no external funding was received. 


\section{References}

[1] R.F. Jacob, The traditional therapeutic paradigm: complete denture therapy, J Prosthet Dent 79 (1998) 6-13.

[2] N. Kawahata, H. Ono, Y. Nishi, T. Hamano, E. Nagaoka, Trial of duplication procedure for complete dentures by CAD/CAM, J Oral Rehabil 24 (1997) 540-8.

[3] Y. Maeda, M. Minoura, S. Tsutsumi, M. Okada, T. Nokubi, A CAD/CAM system for removable denture. Part I: Fabrication of complete dentures, Int J Prosthodont 7 (1994) 1721.

[4] N.Z. Baba, H.S. AlRumaih, B.J. Goodacre, C.J. Goodacre, Current techniques in CAD/CAM denture fabrication, Gen Dent 64 (2016) 23-8.

[5] H. Chen, H. Wang, P. Lv, Y. Wang, Y. Sun, Quantitative Evaluation of Tissue Surface Adaption of CAD-Designed and 3D Printed Wax Pattern of Maxillary Complete Denture, Biomed Res Int 2015 (2015) 453968.

[6] B.J. Goodacre, C.J. Goodacre, N.Z. Baba, M.T. Kattadiyil, Comparison of denture base adaptation between CAD-CAM and conventional fabrication techniques, J Prosthet Dent 116 (2016) 249-56.

[7] M. Inokoshi, M. Kanazawa, S. Minakuchi, Evaluation of a complete denture trial method applying rapid prototyping, Dent Mater J 31 (2012) 40-6.

[8] M. Srinivasan, Y. Cantin, A. Mehl, H. Gjengedal, F. Muller, M. Schimmel, CAD/CAM milled removable complete dentures: an in vitro evaluation of trueness, Clin Oral Investig 21 (2017) 2007-19.

[9] O. Steinmassl, H. Dumfahrt, I. Grunert, P.A. Steinmass1, CAD/CAM produces dentures with improved fit, Clin Oral Investig (2018) doi: 10.1007/s00784-018-2369-2. [Epub ahead of print]. 
[10] M. Srinivasan, H. Gjengedal, M. Cattani-Lorente, M. Moussa, S. Durual, M. Schimmel, F. Müller, CAD/CAM milled complete removable dental prostheses: an invito evaluation of biocompatibility, mechanical properties, and surface roughness, Dent Mater J 37 (2018) 52633.

[11] O. Steinmassl, H. Dumfahrt, I. Grunert, P.A. Steinmassl, Influence of CAD/CAM fabrication on denture surface properties, J Oral Rehabil 45 (2018) 406-13.

[12] A.S. Bidra, K. Farrell, D. Burnham, A. Dhingra, T.D. Taylor, C.L. Kuo, Prospective cohort pilot study of 2-visit CAD/CAM monolithic complete dentures and implant-retained overdentures: Clinical and patient-centered outcomes, J Prosthet Dent 115 (2016) 578-86.

[13] M.T. Kattadiyil, R. Jekki, C.J. Goodacre, N.Z. Baba, Comparison of treatment outcomes in digital and conventional complete removable dental prosthesis fabrications in a predoctoral setting, J Prosthet Dent 114 (2015) 818-25.

[14] N.M. Pereyra, J. Marano, G. Subramanian, S. Quek, D. Leff, Comparison of Patient Satisfaction in the Fabrication of Conventional Dentures vs. DENTCA (CAD/CAM) Dentures: A Case Report, J N J Dent Assoc 86 (2015) 26-33.

[15] M. Schimmel, Y. Cantin, M. Srinivasan, F. Müller, [The Digital Denture in the Clinical Test], Teamwork: J Cont Dent Educ. 19 (2016) 216-30.

[16] M.T. Kattadiyil, C.J. Goodacre, N.Z. Baba, CAD/CAM complete dentures: a review of two commercial fabrication systems, J Calif Dent Assoc 41 (2013) 407-16.

[17] F. Tasker, How to lead a quality improvement project., British Medical Journal 343 (2013) f113.

[18] J. Schreinemakers, Die Logik in der Totalprothetik, Quintessenz, Berlin, 1979.

[19] F. Faul, E. Erdfelder, A. Buchner, A.G. Lang, Statistical power analyses using G*Power 3.1: tests for correlation and regression analyses, Behavior research methods 41 (2009) 114960. 
[20] Y. Kawai, H. Murakami, Y. Takanashi, J.P. Lund, J.S. Feine, Efficient resource use in simplified complete denture fabrication, Journal of prosthodontics : official journal of the American College of Prosthodontists 19 (2010) 512-6. 
Table 1. Comparison of the two complete denture protocols in terms of clinical chairside time and costs (clinical material and laboratory).

Clinical chairside time spent [ minutes (†hours)]

\begin{tabular}{|c|c|c|c|c|}
\hline \\
\hline Group & $\mathrm{CCDP}($ mean time $\pm \mathrm{SD})$ & DDP (mean time \pm SD) & Difference & p-value \\
\hline$\# 1$ - Upper CD & $435.8 \pm 74.1^{\sim}(7.3 \pm 1.2)^{\dagger}$ & $327.5 \pm 34.6^{\sim}(5.5 \pm 0.6)^{\dagger}$ & $108.3 \pm 79.5^{\sim}(1.8 \pm 0.4)^{\dagger}$ & $0.0206^{*}$ \\
\hline \#2 - Upper \& Lower CD & $644.0 \pm 54.1^{\sim}(10.7 \pm 0.9)^{\dagger}$ & $411.0 \pm 36.0^{\sim}(6.9 \pm 0.6)^{\dagger}$ & $233.0 \pm 72.7^{\sim}(3.7 \pm 2.1)^{\dagger}$ & $0.0020 *$ \\
\hline \multicolumn{5}{|c|}{ Clinical materials cost (Swiss francs) } \\
\hline Group & $\mathrm{CCDP}($ mean cost $\pm \mathrm{SD})$ & $\mathrm{DDP}($ mean cost $\pm \mathrm{SD})$ & ssp:Difference & $\mathrm{p}$-value \\
\hline$\# 1$ - Upper CD & $11.50 \pm 5.27$ & $170.70 \pm 16.88$ & $159.19 \pm 15.04$ & $<0.0001^{*}$ \\
\hline$\# 2$ - Upper \& Lower CD & $18.46 \pm 1.91$ & $202.79 \pm 29.55$ & $184.33 \pm 30.64$ & $0.0002^{*}$ \\
\hline \multicolumn{5}{|c|}{ Laboratory costs (in Swiss francs) } \\
\hline Group & $\mathrm{CCDP}($ mean cost $\pm \mathrm{SD})$ & $\mathrm{DDP}($ mean cost $\pm \mathrm{SD})$ & Sspifference & $\mathrm{p}$-value \\
\hline$\# 1$ - Upper CD & $942.67 \pm 113.18$ & $517.56 \pm 0.00$ & $425.11 \pm 113.18$ & $0.0003^{*}$ \\
\hline \#2 - Upper \& Lower CD & $1980.8 \pm 504.94$ & $819.91 \pm 61.78$ & $1160.89 \pm 445.19$ & $0.0043 *$ \\
\hline \multicolumn{5}{|c|}{ Overall costs for clinical materials + laboratory fees (Swiss francs) } \\
\hline Group & $\mathrm{CCDP}($ mean cost $\pm \mathrm{SD})$ & $\mathrm{DDP}($ mean cost $\pm \mathrm{SD})$ & Isp:Difference & $\mathrm{p}$-value \\
\hline$\# 1$ - Upper CD & $954.17 \pm 110.45$ & $688.26 \pm 16.88$ & $265.91 \pm 123.08$ & $0.0032 *$ \\
\hline \#2 - Upper \& Lower CD & $1999.26 \pm 505.39$ & $1022.70 \pm 74.09$ & $976.56 \pm 444.84$ & $0.0080^{*}$ \\
\hline
\end{tabular}


Table 2. Cost minimization analysis.

\begin{tabular}{|c|c|c|c|}
\hline & $\operatorname{CCDP}^{(A)}$ & $\mathbf{D D P}^{(\mathbf{B})}$ & Difference $^{(A-B)}$ \\
\hline Clinical costs charged to the patient $(\mathrm{CHF})^{(\mathrm{a})}$ & $* 1367.10$ & $* 1367.10$ & 0.00 \\
\hline Average laboratory fees (CHF) & 1980.80 & 819.91 & 1160.89 \\
\hline Total charges for the patient (CHF) & 3347.90 & 2187.01 & 1160.89 \\
\hline Mean clinical materials charges $(\mathrm{CHF})^{(\mathrm{b})}$ & 18.46 & 202.79 & -184.33 \\
\hline Mean chairside time (hours) ${ }^{(\mathrm{c})}$ & 10.73 & 6.85 & 3.88 \\
\hline Institution's estimated labour costs $(\mathrm{CHF})^{(\mathrm{d}=\mathrm{a}-\mathrm{b})}$ & 1348.64 & 1164.31 & 184.33 \\
\hline Estimated hourly labour costs $(\mathrm{CHF})^{(\mathrm{d} / \mathrm{c})}$ & 125.69 & 169.97 & -44.28 \\
\hline Adjusted estimated labour costs $(\mathrm{CHF})^{\dagger}$ & 1348.64 & 860.98 & 487.66 \\
\hline $\begin{array}{l}\text { Projected institutional profits in the next } 10 \text { years if DDP was employed } \\
(\mathrm{CHF})^{* *}\end{array}$ & - & 146298.00 & - \\
\hline
\end{tabular}




\section{TABLE LEGENDS}

Table 1. Comparison of the two complete denture protocols in terms of clinical chairside time and costs (clinical material and laboratory).

Table 2. Cost minimization analysis. 